

\title{
Alternativas Ecológicas de Control de Mycosphaerella fijiensis en el Cultivo de Plátano
}

\author{
Juan Carlos Nava ${ }^{1} *$ D, Tibisay Coromoto Urdaneta ${ }^{1}$ (i) y Paulo Humberto \\ Centanaro $^{2}$ (iD \\ ${ }^{1}$ Departamento de Agronomía, Facultad de Agronomía, Universidad del Zulia. Maracaibo 4005, \\ Venezuela. \\ ${ }^{2}$ Universidad Agraria del Ecuador, Provincia de Guayas 090104, Ecuador. \\ *Autor de correspondencia: navauniversidaddelzulia@gmail.com \\ https://doi.org/10.22209/rt.v44n3a06
}

Recepción: 01 de diciembre de 2020 | Aceptación: 09 de julio de 2021 | Publicación: 01 de agosto 2021

\section{Resumen}

El plátano es un cultivo de gran importancia para el desarrollo social de muchas regiones, pero se requiere un cambio, orientando hacia la protección ambiental, utilizando diferentes alternativas naturales. De esta manera, el objetivo de la investigación fue: evaluar alternativas ecológicas para el control de Sigatoka negra (Mycosphaerella fijiensis Morelet) en el cultivo de plátano (Musa AAB) en la zona del sur del Lago de Maracaibo. Los tratamientos fueron: T1: testigo sin ningún tipo de aplicación de producto; T2: lixiviado de raquis de plátano; T3: vermicompost; T4: sulfa-Ca-Mg (caldo sulfocálcico); T5: dióxido de cloro (BioAus) y T6: Propizole 250 CE. Los datos fueron analizados utilizando la metodología de medidas repetidas a fin de evaluar las variables en estudio a través del tiempo, ajustando un modelo polinómico de segundo grado. Es viable la utilización de productos de origen biológico como alternativa ecológica para el manejo de $M$. fijiensis en plantaciones de plátano, a fin de disminuir el impacto negativo de los agroquímicos. Con aplicaciones semanales de alguna de las alternativas ecológicas, se podría mantener la hoja número tres como la más joven que presentó la enfermedad y grado tres o cuatro de incidencia de la misma.

Palabras clave: agroquímicos; control biológico; Musa AAB; Sigatoka negra.

\section{Ecological Control Alternatives for Mycosphaerella fijiensis in Plantain Crop}

\begin{abstract}
Plantain is a crop of big importance for the social development of many regions, but a change oriented towards the environmental protection using several natural alternatives is required. In this way, the objective of this research was: to evaluate ecological alternatives for the control of black Sigatoka (Mycosphaerella fijiensis Morelet) in plantain crop (Musa AAB) in the southern area of Lago de Maracaibo. The treatments were: T1: control without any type of product application; T2: plantain rachis leachate; T3: vermicompost; T4: sulpha-Ca-Mg (sulfocalcic broth); T5: chlorine dioxide (BioAus) and T6: Propizole 250 EC. The data were analyzed using the repeated measures methodology in order to evaluate the variables under study over time, adjusting a second-grade polynomial model. The use of biological products is viable as an ecological alternative for the management of $M$. fijiensis on plantain plantations, with the purpose to decrease the negative impact of agrochemicals. With weekly applications of some of the ecological alternatives, the leave number three could be maintained as the youngest leaf that presented the disease and grade three or four of the incidence of the same.
\end{abstract}

Key words: agrochemicals; biological control; black Sigatoka; Musa AAB. 


\section{Introducción}

El plátano es un rubro de gran importancia en lo social, alimentario y económico; siendo un producto característico en la dieta de muchas personas, por el consumo y los aportes nutricionales que ofrece; es un alimento rico en vitamina $\mathrm{C}$, potasio, fósforo, hierro, magnesio, entre otros (Nava, 2019a). Los plátanos y bananos son de gran importancia en los países en vías de desarrollo. Más de 40 millones de personas que habitan en los trópicos y subtrópicos dependen del cultivo de las musáceas; significando un ingreso económico relevante para muchos países productores, al comercializarse tanto en los mercados locales como en los mercados de exportación, y constituyen una importante fuente de empleo en numerosos países en desarrollo (PACAZ, 2008). En este sentido, el 72,7 \% de la producción de plátano se concentra en África, 22,9 \% en América y 4,4 \% en Asia. La mayoría de la producción mundial se destina para consumo interno en cada país, sin embargo, varios países exportan este fruto (FAOSTAT, 2017).

El plátano es de gran importancia en la alimentación familiar, generando ingresos permanentes para pequeños y medianos agricultores, además de aportar al desarrollo social de muchas regiones; el fruto constituye un alimento básico en la dieta, tanto para la población urbana como para el sector rural (Marrufo et al., 2015). La producción de plátano en Venezuela es de gran importancia socioeconómica desde el punto de vista de seguridad alimentaria y la generación de empleo. Los principales centros productores de plátano, se encuentran concentrados en la zona sur del Lago de Maracaibo (Zulia, Trujillo y Mérida), lo que tiene particular importancia debido a las condiciones agroecológicas existentes; clima tropical, riqueza del suelo y la tradición que se mantiene por herencia familiar (Hernández y Vit, 2013).

La Sigatoka negra (SN, causada por Mycosphaerella fijiensis); es muy importante y limitante en la producción de las musáceas a nivel mundial; afecta el área foliar fotosintética de las plantas y en consecuencia, los racimos y los frutos presentan una menor biomasa (Álvarez et al., 2013). Al respecto, se debe conocer la enfermedad, sintomatología, importancia, manejo y monitoreo, para poder evaluarla en campo, con prácticas culturales oportunas, entre otros, bajo la supervisión constante, donde se establezcan programas de control adecuados para disminuir el grave deterioro ambiental y social causado por la aplicación continua e indiscriminada de agroquímicos (Nava et al., 2017).

Los nuevos conocimientos y las nuevas realidades del entorno requieren un cambio de enfoque, orientando hacia la optimización en las unidades productivas del uso de los recursos internos, externos y la protección ambiental; tal enfoque pretende satisfacer las necesidades actuales de la población sin comprometer el ambiente, utilizando las diferentes alternativas naturales que existen y que pueden ser producidas en cada unidad de producción, antes de utilizar un determinado tipo de agroquímico (Nava, 2019b). En este contexto, se conocen diferentes productos para tales fines, entre los que se encuentran: i) el lixiviado de raquis, es un abono orgánico producido a partir de la deshidratación del raquis del racimo de plátano; es usado por los agricultores como abono orgánico líquido, por ser rico en potasio y micro elementos, al mismo tiempo contiene gran cantidad de microorganismos, considerados como pesticidas (Hernández, 2009); ii) el vermicompost, es producto de la descomposición de materia orgánica, realizada únicamente por ciertas especies de lombrices, específicamente del género Eisenia, la cual realiza un proceso de descomposición más rápidamente, transformando la materia orgánica en lo que se denomina humus de lombriz o vermicompost (Alarcón, 2005); iii) el caldo sulfocálcico, es una mezcla de azufre en polvo y cal; su preparación consiste en poner a hervir en agua durante $1 \mathrm{~h}$. El caldo debe de tener una apariencia amarillenta o anaranjada (Núñez et al., 2003); iv) el dióxido de cloro, es la mezcla de clorito de sodio y algún ácido débil (comúnmente ácido cítrico). Elimina microorganismos por la interrupción del transporte de nutrientes a través de la membrana celular; siendo un oxidante selectivo, eficaz, no corrosivo (Deininger et al., 2000); y v) el Propizole 250 CE, es un fungicida; cuyo ingrediente activo es propiconazole, del grupo químico de los triazoles; con una concentración de $250 \mathrm{~g}$ de ingrediente activo por litro de producto y es compatible con otros fungicida de uso común, siendo ligeramente toxico (Fraga, 2008).

Ahora bien, el gran potencial de las zonas productoras de plátano se encuentra amenazado significativamente, debido, en gran parte, por consecuencias de las altas precipitaciones, alta humedad relativa, mal manejo de plantaciones y uso inadecuado de fungicidas químicos, lo cual favorece la proliferación de esporas de $M$. fijiensis (Nava, 2002), lo que ha llevado a la necesidad de probar nuevas alternativas como fungicidas biológicos y mejorar los planes de manejo, para controlar dicha enfermedad por parte de los productores. Es importante que el productor de plátanos conozca las diferentes alternativas para el manejo de la $\mathrm{SN}$, con la finalidad de mejorar las 
condiciones y minimizar el impacto negativo en los seres humanos, animales y el ambiente, buscando mantener un equilibrio. De esta manera, el objetivo de la investigación fue, evaluar alternativas ecológicas para el control de $M$. fijiensis en el cultivo de plátano en el sur del Lago de Maracaibo.

\section{Materiales y Métodos}

\section{Condiciones experimentales}

La investigación se llevó a cabo en la región sur del Lago de Maracaibo, municipio Colón del estado Zulia, parroquia Santa Bárbara del Zulia, en la carretera Santa Bárbara - Cuatro Esquinas, entre el sector El Manguito y el sector Guaimaral, unidad de producción El Porvenir; con una zona de vida de bosque húmedo tropical y bosque muy húmedo tropical con una precipitación media de $2.200 \mathrm{~mm} / \mathrm{año}$; y una evaporación media anual de $1.415 \mathrm{~mm}$. En cuanto a la temperatura, se registra una media de $30{ }^{\circ} \mathrm{C}$ con una altitud de 200 a $500 \mathrm{msnm}$ y una humedad relativa del 70 - $80 \%$. La región presenta suelos correspondientes a una planicie aluvial de origen reciente, con alta fertilidad, textura mediana, franco, franco-arcilloso, franco-arcillo-limoso, franco-limoso y un $\mathrm{pH} 7,1-8,2$ (CORPOZULIA, 2018).

\section{Material experimental}

Se evaluó una plantación de plátano Hartón; de aproximadamente 15 meses de establecida, con una distancia de siembra de $3 \mathrm{~m}$ entre hileras x $2 \mathrm{~m}$ entre plantas, para un total de 1.667 plantas/ha. En total se evaluaron 180 plantas, realizando semanalmente labores de cirugía y deshoje; para evaluar la incidencia y severidad de la enfermedad, utilizando la escala de Stover (Nava, 2002). Se registró el grado de incidencia de la enfermedad, total de hojas en la planta (NHP) y hoja más joven infectada (HMJ) (Tabla 1).

Tabla 1. Grados de incidencia para evaluar la severidad de M. fijiensis según la escala de Stover (Nava, 2002).

\begin{tabular}{cc}
\hline Grado de incidencia & Intensidad de ataque o severidad \\
\hline 0 & $0 \%$ (sana). \\
1 & Hasta 10 pizcas. \\
2 & $1-5 \%$ área foliar afectada. \\
3 & $6-15 \%$ área foliar afectada. \\
4 & $16-33 \%$ área foliar afectada. \\
5 & $34-50 \%$ área foliar afectada. \\
6 & $>50 \%$ área foliar afectada. \\
\hline
\end{tabular}

\section{Unidad experimental}

La unidad experimental fue la planta de plátano variedad Hartón, género Musa.

\section{Tratamientos}

En la Tabla 2 se muestran los seis tratamientos del ensayo, testigo, sin ningún tratamiento, lixiviado de raquis, vermicompost, caldo sulfocálcico (sulfa-Ca-Mg), dióxido de cloro (BioAus) y propizole $250 \mathrm{CE}$.

Tabla 2. Tratamiento y características de las alternativas ecológicas de control de $M$. fijiensis en el cultivo del plátano Hartón.

\begin{tabular}{cl}
\hline Tratamientos & \multicolumn{1}{c}{ Características } \\
\hline 1 & Testigo, sin aplicación de algún producto. \\
2 & Lixiviado de raquis de plátano, aplicado $100 \%$ o puro. \\
3 & Vermicompost, 25 L por cada $100 \mathrm{~L}$ de agua. \\
4 & Sulfa-Ca-Mg (caldo sulfocálcico), 40 L por cada $100 \mathrm{~L}$ de agua. \\
5 & Producto biológico BioAus (dióxido de cloro), 60 L por cada $100 \mathrm{~L}$ de agua. \\
6 & $\begin{array}{l}\text { Testigo con producto químico Propizole } 250 \mathrm{CE} ; 0,1 \mathrm{~L} \text { por cada } 100 \mathrm{~L} \text { de } \\
\text { agua. }\end{array}$ \\
*Dosis recomendada por el fabricante.
\end{tabular}




\section{Procedimiento experimental}

En la unidad de producción se identificaron 30 plantas por tratamiento, delimitando el área con cintas de diferentes colores; se seleccionaron plantas que estaban deshojadas y sin emisión de la bellota, con 18 semanas de edad. Se hizo una aplicación de cada uno de los tratamientos mencionados en la semana correspondiente, usando una asperjadora de espalda tipo mochila.

La evaluación de la enfermedad se hizo por 35 d, calificando el daño de área foliar en grados según la escala de Stover, a partir de la hoja más joven infectada HMJI. En campo se contó el número total de hojas NHP en las plantas seleccionadas, luego se observó la HMJ, se anotó el grado para cada repetición y tratamiento, desde la primera evaluación hasta la última toma de datos.

\section{Diseño experimental}

El diseño experimental fue de bloques completamente al azar con seis tratamientos y treinta repeticiones. Los datos fueron analizados usando la metodología de medidas repetidas a fin de evaluar las variables en estudio a través del tiempo, ajustando el siguiente modelo polinómico de segundo grado, con la Ecuación 1:

$$
Y=a+b x+c x^{2}
$$

Donde: a, b y c: parámetros del modelo, $\mathrm{X}$ : efecto lineal en el tiempo, $\mathrm{X}^{2}$ : efecto cuadrático en el tiempo.

\section{Variables evaluadas}

El número de hojas en la planta (NHP), se entiende como el número total de hojas funcionales y que se puedan contabilizar en una planta. La hoja más joven infectada con SN (HMJI), corresponde a la primera hoja totalmente abierta que presentó hasta 10 pizcas, es decir grado 1, contando las hojas de arriba hacia abajo. El grado de incidencia de la enfermedad (GIE), hace referencia a la incidencia de la enfermedad en la extensión del área de la hoja infectada por el patógeno, expresándose en grados de la enfermedad (Nava, 2019a).

\section{Técnicas de procesamiento y análisis de datos}

Para el análisis de los datos recolectados, se utilizó la metodología de medidas repetidas en el tiempo; ajustando un polinomio de segundo grado, a través del procedimiento PROC MIXED del paquete estadístico SASß (SAS, 2020), utilizando el programa Microsoft Office Excel 2010 para elaborar la base de datos y elaboración de figuras.

\section{Resultados y Discusión}

\section{Número de hojas por planta}

El análisis estadístico generó modelos matemáticos de segundo orden para estimar la respuesta de la variable NHP, como se observa en la Tabla 3 , donde $\mathrm{X}$ representa el efecto lineal en el tiempo y $\mathrm{X}^{2}$ el efecto cuadrático en el tiempo.

Tabla 3. Modelo matemático de segundo orden para estimar la respuesta del número de hojas por planta (NHP) en el cultivo del plátano.

\begin{tabular}{cc}
\hline Tratamiento & Modelo \\
\hline T1 & $6,463+0,115 \mathrm{X}-0,0019 \mathrm{X}^{2}$ \\
T2 & $6,573+0,125 \mathrm{X}-0,0022 \mathrm{X}^{2}$ \\
T3 & $6,410+0,101 \mathrm{X}-0,0017 \mathrm{X}^{2}$ \\
T4 & $6,091+0,087 \mathrm{X}-0,0014 \mathrm{X}^{2}$ \\
T5 & $6,053+0,081 \mathrm{X}-0,0012 \mathrm{X}^{2}$ \\
T6 & $5,309+0,128 \mathrm{X}-0,0019 \mathrm{X}^{2}$ \\
\hline X: efecto lineal en el tiempo, $\mathrm{X}^{2}$ : efecto cuadrático en el tiempo.
\end{tabular}


El NHP de plátano se estimó entre 7 y 8 hojas. Para T1, T2 y T3 la estimación fue de 7 hojas por planta, mientras que en T4, T5 y T6 fue de 8 hojas por plantas (Figura 1).

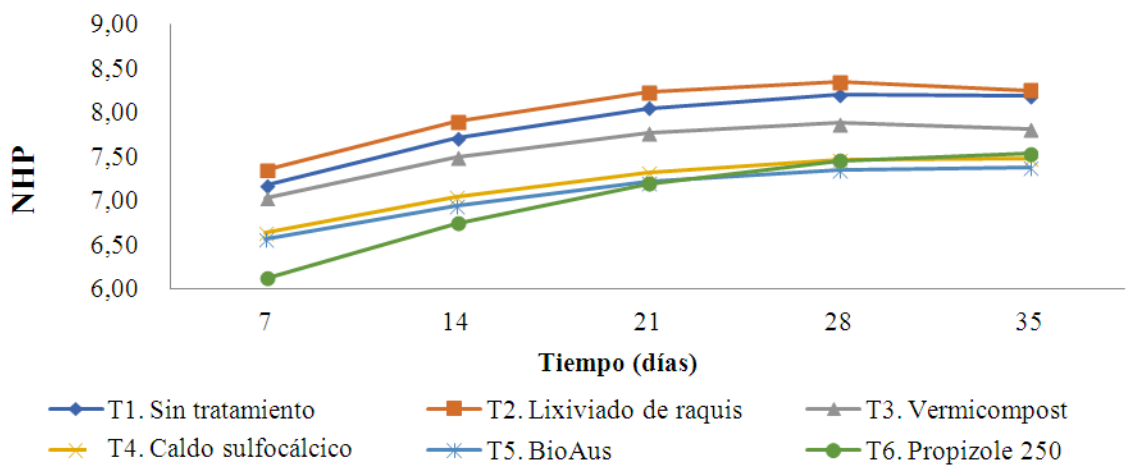

Figura 1. Estimación a través del tiempo del número de hojas por planta (NHP) en el cultivo de plátano.

En el rubro plátano, un número medio de 8 hojas; es suficiente para obtener racimos con buena biomasa y calidad (Güerere et al, 2008; Marín et al., 2008); lo cual coincide con los resultados obtenidos en esta investigación. Aunque el número de hojas que puede producir la planta durante su ciclo es variado, es importante que la planta presente un número adecuado de hojas fotosintéticamente activas. Las estimaciones para la variable NHP se incrementan a medida que pasa el tiempo, siendo esto favorable en la planta de plátano para un adecuado llenado de los frutos en el racimo. El número de hojas que presenta la planta durante su ciclo de vida es de gran importancia para garantizar la productividad y calidad de los frutos (Ramírez et al., 2014).

\section{Hoja más joven infectada}

El análisis estadístico generó modelos matemáticos de segundo orden para estimar la respuesta de la variable HMJ, como se observa en la Tabla 4, donde $\mathrm{X}$ representó el efecto lineal en el tiempo y $\mathrm{X}^{2}$ el efecto cuadrático en el tiempo.

Tabla 4. Modelo matemático de segundo orden para estimar las respuestas de la hoja más joven infectada (HMJI).

\begin{tabular}{cc}
\hline Tratamiento & Modelo \\
\hline T1 & $3,4900+0,01053 \mathrm{X}-0,00079 \mathrm{X}^{2}$ \\
T2 & $3,7367-0,01485 \mathrm{X}-0,00026 \mathrm{X}^{2}$ \\
T3 & $3,4467-0,00939 \mathrm{X}-0,00029 \mathrm{X}^{2}$ \\
T4 & $3,6204+0,03185 \mathrm{X}-0,00126 \mathrm{X}^{2}$ \\
T5 & $2,7167+0,1021 \mathrm{X}-0,00251 \mathrm{X}^{2}$ \\
T6 & $3,1961+0,04855 \mathrm{X}-0,00129 \mathrm{X}^{2}$ \\
\hline
\end{tabular}

Como se observa en la Figura 2, en T4, T5 y T6 las estimaciones mantuvieron la hoja 3 como la HMJI hasta 28 d después de la aplicación, para T1, T2 y T3; las estimaciones mantuvieron la hoja 2 como la HMJI a los 28 d después de la aplicación. Durante los primeros siete días todos los tratamientos mantuvieron la hoja 3 como la más joven, por lo que se podrían realizar aplicaciones semanales de todos los extractos estudiados. 


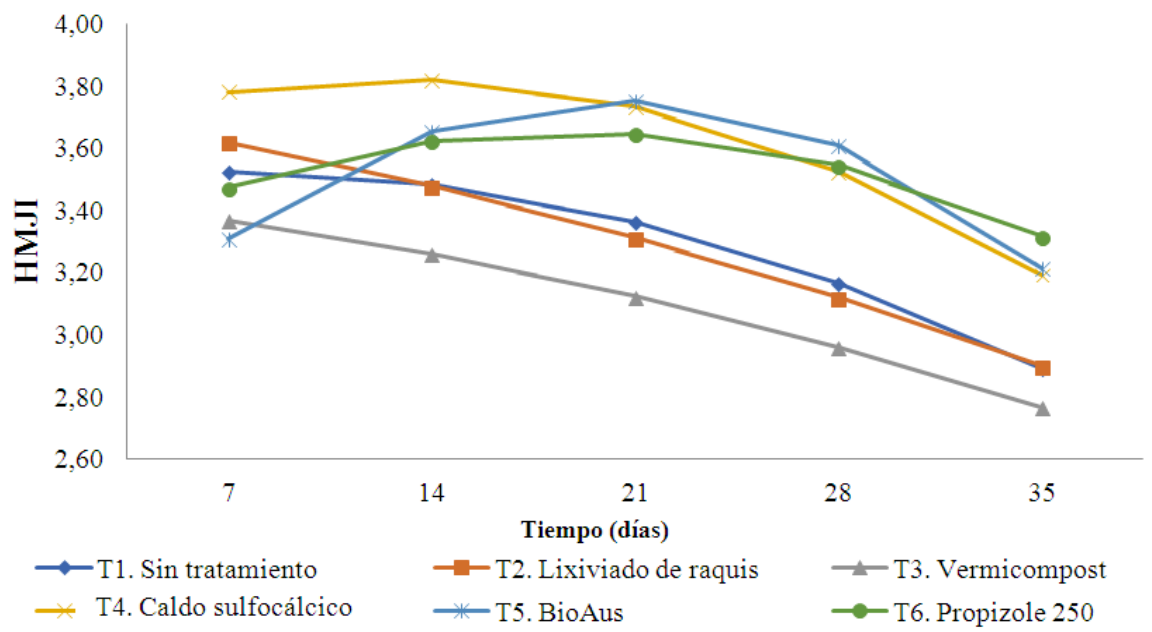

Figura 2. Estimación a través del tiempo de la hoja más joven infectada (HMJ) de M. fijiensis en el cultivo de plátano.

Las hojas infectadas representan la única fuente de inóculo de la enfermedad, por lo que debe realizarse un manejo adecuado para disminuir la esporulación del patógeno a través del tiempo (Noa et al., 2018). En este contexto, los productores deben evaluar sus unidades productivas de plátano para verificar el estado fitosanitario de las plantas con respecto a la enfermedad, para prevenir daños severos al cultivo y tener una producción adecuada (Ramírez et al., 2014).

La enfermedad no se debe manejar como una actividad aislada, sino como un conjunto de prácticas y acciones coordinadas en el tiempo, con la supervisión constante como un gran protagonista, por lo tanto, se considera necesario que en las diferentes unidades productivas se revise semanalmente el avance de la enfermedad, determinando la hoja más joven que se encuentra infectada, para analizar la situación, tomar acciones necesarias sobre las prácticas realizadas y productos aplicados, y planificar las próximas actividades a realizar. Con la opción de diferentes alternativas ecológicas, se pueden establecer un programa de control adecuado para disminuir el grave deterioro ambiental y social causado por la aplicación continua e indiscriminada de agroquímicos.

\section{Grado de incidencia de la enfermedad}

El análisis estadístico generó modelos matemáticos de segundo orden para estimar la respuesta de la variable GIE, como se observa en la Tabla 5, donde $\mathrm{X}$ representa el efecto lineal en el tiempo y $\mathrm{X}^{2}$ el efecto cuadrático en el tiempo.

Tabla 5. Modelo matemático de segundo orden para estimar la respuesta del grado de incidencia de la enfermedad (GIE).

\begin{tabular}{cc}
\hline Tratamiento & Modelo \\
\hline T1 & $2,8133+0,1678 \mathrm{X}-0,00258 \mathrm{X}^{2}$ \\
T2 & $2,8367+0,1962 \mathrm{X}-0,00324 \mathrm{X}^{2}$ \\
T3 & $2,0067+0,2251 \mathrm{X}-0,000347 \mathrm{X}^{2}$ \\
T4 & $0,8900+0,2712 \mathrm{X}-0,00393 \mathrm{X}^{2}$ \\
T5 & $0,7733+0,2586 \mathrm{X}-0,00355 \mathrm{X}^{2}$ \\
T6 & $2,8645+0,06947 \mathrm{X}-0,00034 \mathrm{X}^{2}$ \\
\hline X: efecto lineal en el tiempo, $\mathrm{X}^{2}$ : efecto cuadrático en el tiempo.
\end{tabular}

En cuando al GIE, se pudo estimar que para todos los tratamientos se mantuvo en grado 4 o menos, durante los primeros siete d; a los $14 \mathrm{~d}$ T1, T2 y T3 mostraron un incremento, como se observa en la Figura 3, por lo que se podrían realizar aplicaciones semanales de cualquier extracto estudiado o aplicaciones cada $14 \mathrm{~d}$ de T4 y T5. 


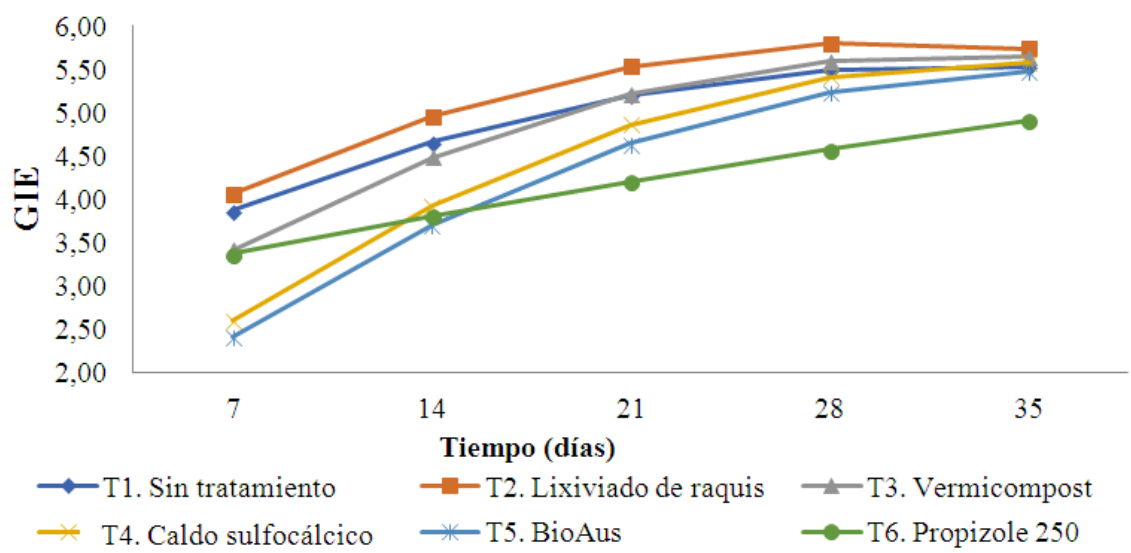

Figura 3. Estimación a través del tiempo de grado de incidencia (GIE) de M. fijiensis en el cultivo de plátano.

Según Barrera et al. (2016) es importante evaluar el grado de incidencia durante cada periodo, determinando la reducción de la severidad de M. fijiensis. Se debe lograr un grado de incidencia bajo, garantizando hojas sanas al momento de floración y cosecha (Morales et al., 2011). Actualmente, existen fungicidas altamente efectivos para el control de la SN; sin embargo, el patógeno ha logrado desarrollar resistencia a algunos de estos productos, poniendo de manifiesto la necesidad de reducir la dependencia del control químico (Lazo et al., 2012). Además, el manejo de la SN debe seguir enfocado en la integración de procedimientos culturales. Los cambios en la percepción de los consumidores de productos más sanos y la preocupación pública por detener la contaminación ambiental, tendrán una influencia marcada en las tecnologías de control que se implementan (Cedeño et al., 2021).

Finalmente, hay que tener en cuenta que para el control de $M$. fijiensis en plátano se utiliza gran cantidad de agroquímicos, que afectan la salud de los humanos, el ambiente y pueden generar poblaciones resistentes del patógeno. Alternativamente, diferentes extractos pueden ser utilizados para el control de dicho patógeno en una producción limpia y sostenible del rubro plátano. En este sentido, todos los extractos estudiados en este trabajo lograron un adecuado control de M. fijiensis, siendo una alternativa de control por un periodo de tiempo de siete d consecutivos después de su aplicación, y 14 d consecutivos para el caso del caldo sulfocálcico y el dióxido de cloro.

\section{Conclusiones}

Todos los productos de origen biológico estudiados representan una alternativa ecológica para el manejo de M. fijiensis en plantaciones de plátano Hartón, a fin de disminuir el impacto negativo a las personas y al ambiente por el uso de los agroquímicos. Con aplicaciones semanales de los extractos se podría mantener la hoja número tres como la más joven que presentó la enfermedad y de grado tres o cuatro de incidencia de la misma. Para el caldo sulfocálcico y el dióxido de cloro las aplicaciones se pueden realizar cada $14 \mathrm{~d}$ de frecuencia entre ciclos.

\section{Referencias Bibliográficas}

Alarcón, A. (2005). Manual de lombricultura. Santander: Editorial de la Universidad de Santander (UDES).

Álvarez, E., Pantoja, A., Ceballos, G., Gañan, L. (2013). Producción de lixiviado de raquis de plátano en el eje cafetalero de Colombia. Cali: Centro Internacional de Agricultura Tropical (CIAT).

Barrera, J., Barraza, F., Campo, R. (2016). Efecto del sombrío sobre la Sigatoka negra (Mycosphaerella fijiensis Morelet) en el cultivo de plátano cv Hartón (Musa AAB Simmonds). Revista U.D.C.A Actualidad y Divulgación Científica, 19(2), 317-323.

Cedeño, J., Díaz, E., Conde, E., Cervantes, A., Avellán, L., Zambrano, M., Tobar, J., Estévez, S., Sánchez, A. (2021). Evaluación de la severidad de Sigatoka negra (Mycosphaerella fijiensis Morelet) en plátano "Barraganete" 
bajo fertilización con magnesio. Revista Técnica de la Facultad de Ingeniería de la Universidad del Zulia, 44(1), 411 .

CORPOZULIA. (2018). Caracterización de las condiciones climáticas y edáficas del municipio Colón. Dirección de información geográfica y estadística de Venezuela. Maracaibo: Corporación de Desarrollo de la Región Zuliana.

Deininger, R., Ancheta, A., Ziegler, A. (2000). El dióxido de cloro, una alternativa como oxidantes y desinfectante del agua potable. Arbor: Escuela de Salud Pública de la Universidad de Michigan.

FAOSTAT. (2017). Estadísticas de producción del cultivo del plátano. [en línea] disponible en: http://faostat.fao.org/faostat/form/collection/Production.Crops [consulta: 10 octubre 2020].

Fraga, L. (2008). Manual técnico. "Agroisleña”. Caracas: Cronotip impresiones.

Güerere, P., Martínez, L., Fuenmayor, L. (2008). Efecto del deshoje inducido sobre la productividad del plátano (Musa AAB) cv. Hartón y la incidencia de Sigatoka negra (Mycosphaerella fijiensis Morelet). Revista de la Facultad de Agronomía, 25, 636-648.

Hernández, F. (2009). Manual práctico para la producción de abonos orgánicos a partir del cultivo de plátano. Maracaibo: Editorial de La Universidad del Zulia (EDILUZ).

Hernández, L., Vit, P. (2013). El plátano, un cultivo tradicional con importancia nutricional. Revista del Colegio de Farmacéuticos del estado Mérida, II, 14-20.

Lazo, J., Muñoz, J., Escalona, A. (2012). Evaluación experimental del clorotalonil en el control de la Sigatoka negra (Mycosphaerella fijiensis) en plantaciones de plátano (Musa sp. AAB). Bioagro, 24(2), 127-134.

Marín, O., Mass, M., Barrera, J., Robles, J. (2008). Evaluación de extractos vegetales para el control de Mycosphaerella fijiensis en plátano en Tierralta - Córdoba. Temas Agrarios, 13(1), 25-31.

Marrufo, J., Prieto, M., Nava, J., Ortega, J., Bracho, B. (2015). Diagnóstico socioeconómico y técnico de los productores de plátano en el sector Las Vegas del municipio Santa Rita, estado Zulia. Revista de la Facultad de Agronomía, 32, 82-105.

Morales, L., Ullauri, M., Rojas, X. (2011). Evaluación del efecto de extractos vegetales como alternativa de manejo a la Sigatoka negra en el cultivar gran enano (AAA). Centro Agrícola, 38(2), 77-84.

Nava, C. (2002). Las enfermedades del plátano en Venezuela, su control. Maracaibo: Editorial Astro Data S.A.

Nava, J. (2019a). Manejo integral del cultivo del plátano. Maracaibo: Editorial Gráficas Nerio Tip C.A.

Nava, J. (2019b). Uso y manejo responsable de agroquímicos. Lea y entienda la etiqueta. Madrid: Editorial Académica Española.

Nava, J., Sánchez, A., Ortega, J. (2017). Gestión de planificación económica en el cultivo del plátano en el estado Zulia, Venezuela. Revista de la Facultad de Agronomía, 34, 371-396.

Noa, J., Valencia, A., Chávez, V., Jarrillo, J., Flores, N., Córdova, C., Jarillo, S., Montero, R., Escobar, R. (2018). Obtención de lixiviados de raquis de plátano: uso potencial en cultivos. Ciencia UANL, 89, 1-8.

Núñez, J., Sánchez, J., Pliego, G., Vulling, M. (2003). Cartilla de abonos orgánicos y caldos minerales. Toluca: Dirección General del Fondo para el Desarrollo Económico - Subdirección del Fomento Agropecuario.

PACAZ. (2008). Manual de buenas prácticas agrícolas. Programa de asistencia técnica y capacitación agrícola del estado Zulia. Maracaibo: Convenio Gobernación del estado Zulia-Universidad del Zulia-SDA Zulia. 
Ramírez, Y., Perozo, Y., Nava, J., Bracho, B. (2014). Frecuencia del despunte y dos tipos de deshoje en el manejo de la Sigatoka negra en el cultivo del plátano, estado Zulia. Revista de la Facultad de Agronomía, 31, 524-538.

Statistical Analysis System (SAS®) (2020). SAS Studio user's guide: statistics. Version 15.1. Cary: SAS Institute Inc. 


\section{REVISTA TECNICA}

DE LA FACULTAD DE INGENIERIA

UNIVERSIDAD DEL ZULIA

Vol. 44. $\mathbf{N}^{\circ} 3$, Septiembre - Diciembre, 2021

Esta revista fue editada en formato digital y publicada en Agosto 2021, por el Fondo Editorial Serbiluz, Universidad del Zulia. Maracaibo-Venezuela

www.luz.edu.ve

www.serbi.luz.edu.ve

www.produccioncientificaluz.org 